\title{
Stable Reflex-based Walking of Forelimbs of a Bio-inspired Quadruped Robot-Modeled Cheetah
}

\author{
Shogo Nakatsu, Andre Rosendo, Kenichi Narioka and Koh Hosoda
}

\begin{abstract}
In contrast to the high movement adaptability of quadruped animals in many environmental conditions, it is hard for conventional quadruped robots to operate in complex environment conditions. We investigate the adaptability of animals' musculo-skeletal systems, by building a bio-inspired quadruped robot named "Pneupard" which duplicates a feline musculo-skeletal system. In this study, we built Pneupard's forelimb which has 14 active muscles, 4 passive muscles and 8 degrees of freedom (DOF). We propose sole reflex-based control and verify its effectiveness by conducting walking experiments, in which the robot performed stable walking with a twodimensional restriction.
\end{abstract}

\section{INTRODUCTION}

Quadruped animals have high movement adaptability in many environmental conditions, and can run over high terrain, climb over big obstacles, and so on. This high adaptability of quadruped animals has been investigated many observational studies. For example, Goslow et al. [1] and Miller et al. [2][3] investigated the behavior of cats' legs and gait when jumping. English et al. [4][5] investigated muscles' activity for each gait by measuring the myoelectric signals. Whelan [6] conducted experiments on decerebrate cats and investigated central pattern generators (CPG). However, it is very difficult to investigate the extent by which locomotion stability depends on musculo-skeletal systems by performing observational studies.

On the contrary, constructivist studies have been performed to make bio-inspired robots and investigate exercise mechanisms. If robots are made to duplicate animal behavior, we need to consider both the required control as well as their body structures. Brooks [7] proposed the generation of intelligence using an agent that has simpler control and interaction between environments. Osuka [8][9] states the importance of passivity-based control systems. It is important to approach robotics from the perspective of embodied cognitive science. Consequently, adaptive behaviors might not be achieved by cerebral advanced control, but are due to interactions between the simple control of CPGs and reflexes, body, and environment.

Quadruped robots are used in many studies that adopt a bio-inspired control such as CPG. Ito et al. [10] investigated

This work was partially supported by a Grant-in-Aid for Scientific Research(23220004) from the Japanese Ministry of Education, Culture, Sports, Science and Technology.

All authors are with Graduate School of Information Science and Technology, Osaka University, 2-1 Yamada-oka, Suita, Osaka, 565-0871, Japan. nakatsu.shougo@ist.osaka-u.ac.jp

andre.rosendodist.osaka-u.ac.jp

narioka@ist.osaka-u.ac.jp

koh.hosoda@ist.osaka-u.ac.jp the gait transitions by performing simulations using CPG and investigating the consumption of energy. Raibert et al. [11] then used a virtual leg control to successfully develop a quadruped robot that trots and walks on irregular terrain. In addition, some studies use bio-inspired robots that duplicate the musculo-skeletal systems seen in animals. Iida et al. [12] produced a quadruped robot using motors and springs that duplicate flexible joints, and observed a bouncing gait . Sakai et al. [13] produced a quadruped robot using pneumatic muscles and springs. They changed the joint stiffness of the robot's spine and observed the gait transitions between walking and bouncing. Tsujita et al. [14] proposed a gait control system for walking and troting using pneumatic artificial muscles and CPG. Yamada et al. [15] presented a quadruped robot which has 10 active muscles and 10 passive muscles replicated using pneumatic artificial muscles and springs in the whole body. Then, they discussed the importance of bi-articular muscles. However, because the robot's forelimbs have few links and muscles (scapula, semitendinosus, soleus etc.), the duplication of musculo-skeletal system is not sufficient. Although these robots have flexible joints and duplicate mono-articular muscles, they do not have complicated musculo-skeletal systems and high-performance locomotion, as is the case with animals. Ekeberg et al. [16] presented a robot which duplicates the musculo-skeletal system of a cat's hindlimb when walking, using a simple control system. In this study, the robot duplicated the hindlimb only, furthermore, the robot was made to walk only in the simulation, and not in a real environment.

Herein, to investigate the adaptability possessed by animals' musculo-skeletal systems, we discuss the adaptability of animals in real environmental conditions. In order to investigate animals by a method of constructivist approach, we developed a quadruped robot. In our previous studies [17][18][19], we built a bio-inspired quadruped robot named "Pneupard" which duplicates a feline musculo-skeletal system of hindlimbs, and we proposed a sole reflex-based control. Then, we successful made the robot walk on a treadmill. Next, we produced Pneupard's bio-inspired forelimbs, and attempted to use them to walk by using a sole reflex-based control which is applied to the hindlimbs. In this paper, we reveal the design of Pneupard's forelimb and perform a walking experiment with the forelimb on a treadmill. We then discuss our results, after which we conclude the paper and present future works. 


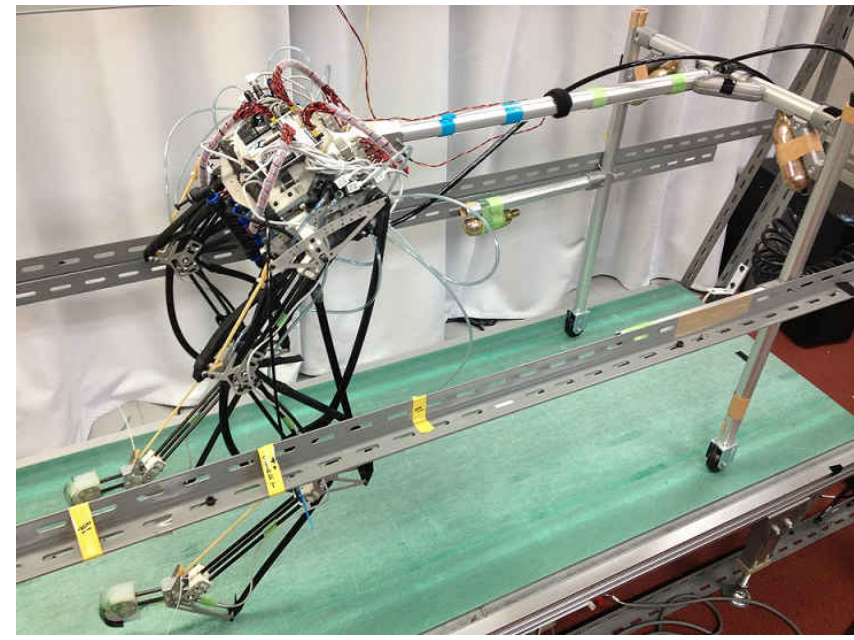

Fig. 1. The design of Pneupard's forelimb. It has four links: scapula, humerus, antebrachial bone, and wrist. Its height is about $700 \mathrm{~mm}$ and weight is about $2.5 \mathrm{~kg}$ without muscles. The spine and hindlimb are connected, and may be regarded as one rigid body. The hindlimb is linked up to guide rails. This is why Pneupard's forelimb cannnot move forward or backward.

\section{The Design of PNeupard's Forelimb}

In this section, we describe the design of Pneupard's forelimb and the proposed control system. Compared with the hindlimb, the forelimb has a more complex structure [18][19]. For example, each hindlimb has 3 links and 3 degree of freedom (DOF), but each forelimb has 4 links and 4 DOF. With regards to the control system, we applied a sole reflex-based system to the forelimb's control. A similar control method has been applied in our previous studies [18][19].

\section{A. The Forelimb Design}

Fig. 1 shows the design of Pneupard's forelimbs. Its link length, range of motion, and moment arm are decided by referring to physiological literature [3][4][5][20]. Each of Pneupard's forelimbs has 4 links (scapula, humerus, antebrachial bone, and carpal) and 4 DOF. Fig. 2 shows the definition of the names of the links and joint angles. Each forelimb has 9 muscles containing the following:(1) monoarticular muscles-: supraspinatus, pectoralis major, triceps brachii (lateral and medial), and flexor carpi ulnaris, and (2) bi-articular muscles: clavo brachialis, biceps brachii, latissimus dorsi, triceps brachii (long), and extensor carpi radialis. Fig. 3 shows the attachment of the muscles. Pneupard's forelimb is made from carbon fiber shafts, ABS plastic, and 2 or $3 \mathrm{~mm}$ thick magnesium plates. The specifications of the robot are shown in Table I. The spine and hindlimbs are connected, and can be regarded as one rigid body. A caster is installed in each hindlimb. Additionally, because the hindlimb is linked up to guide rails, the robot cannot move forward or backward. This is because the forelimb cannot walk on a treadmill when the hindlimb is not linked to the guide rails. We employed Mckibben type pneumatic artificial muscles because pneumatic artificial muscles have not only
TABLE I

KEY OF PNEUPARD’s ForeLIMB

\begin{tabular}{c|c}
\hline Property & Value \\
\hline Body height & $700 \mathrm{~mm}$ \\
\hline Body width & $300 \mathrm{~mm}$ \\
\hline Body weight (Forelimb only) & $3 \mathrm{~kg}$ \\
\hline Body weight (Include hindlimb) & $6.5 \mathrm{~kg}$ \\
\hline No. of degree of freedom & 8 \\
\hline No. of valves & 16 \\
\hline No. of active muscles & 14 \\
\hline No. of passive muscles & 4 \\
\hline No. of total muscles & 18 \\
\hline
\end{tabular}

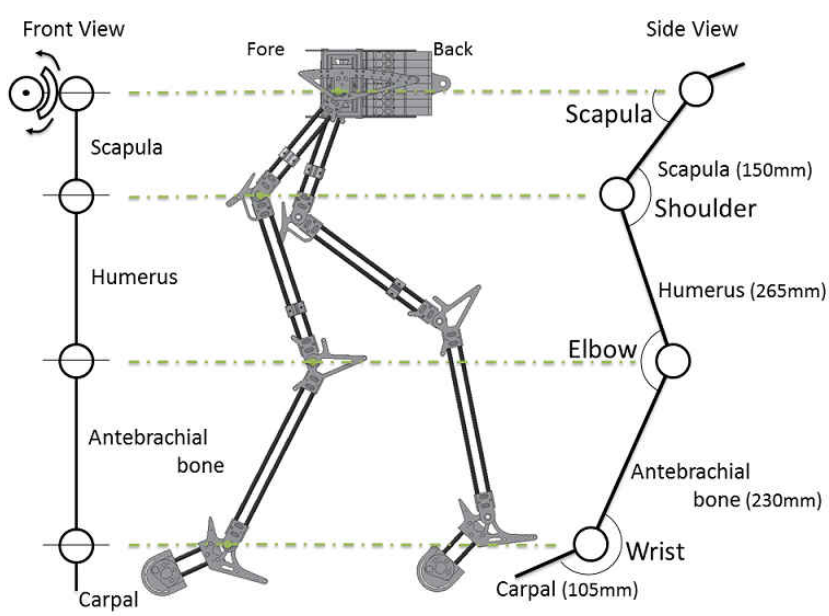

Fig. 2. The definition of names of links and joint angles. The center image is depicts the designed forelimb drawn by $3 \mathrm{D}$ CAD software. The left image shows a front view of forelimb and the right picture shows the side view. Pneupard's forelimb consists of 4 links[scapula, humerus, antebrachial bone and carpal] and has $4 \mathrm{DOF}$.

flexibility and elasticity, which is not easily generated by motor control, but also some of the non-linear properties of biological muscles. If a high air pressure is supplied into a pneumatic muscle, it contracts and hardens. In this process, a pneumatic muscle generates high power. If it releases the air, it returns to its original length and becomes soft again. Pneumatic artificial muscles contract by about $24 \%$. The muscles' moment arm is nearly equal to that of a real cheetah's. Their lengths are decided by considering the range of motion of the joints. Fig. 4 shows a graph comparing the muscles' moment arm.

We think that Pneupard's forelimbs are more complex than other robots as [14][15][21] and more biomimetic. The reason for this is that these robots don't have any bi-articular muscles and scapular mechanism though Pneupard has both of them.

\section{B. Sole Reflex-based Control System}

As is the case with hindlimbs, we propose a sole reflexbased control using FSR (Force Sensing Register) referred by Ekeberg et al. [16]. We divide the walking into four phases: stance, liftoff, swing and touchdown, and make a muscle activity pattern for each phase. Fig. 5 shows the 


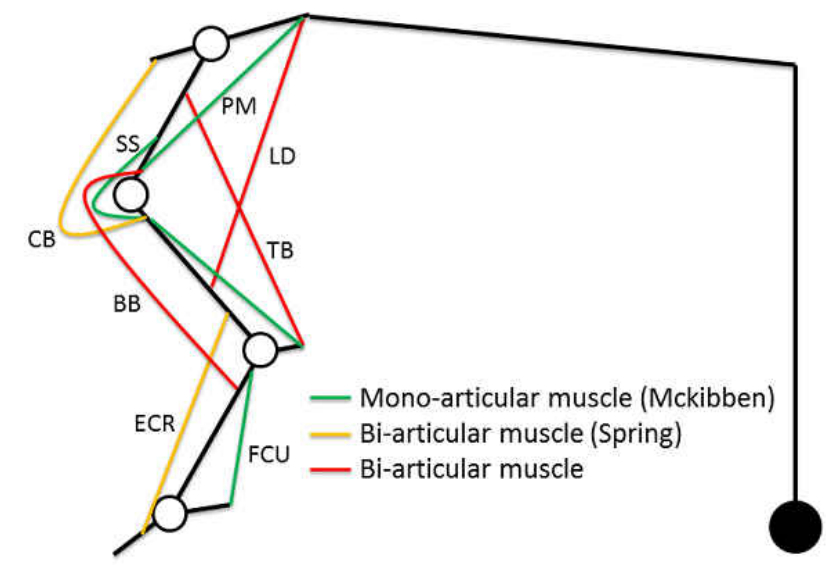

Fig. 3. The muscle arrangement and DOF of Pneupard's forelimb. Each forelimb has 4 DOF, 5 bi-articular muscles [clavo brachialis, biceps brachii, latissimus dorsi, triceps brachii (long), and extensor carpi radialis] and 4 mono-articular muscles [supraspinatus, pectoralis major, triceps brachii (lateral and medial) and flexor carpi ulnaris].

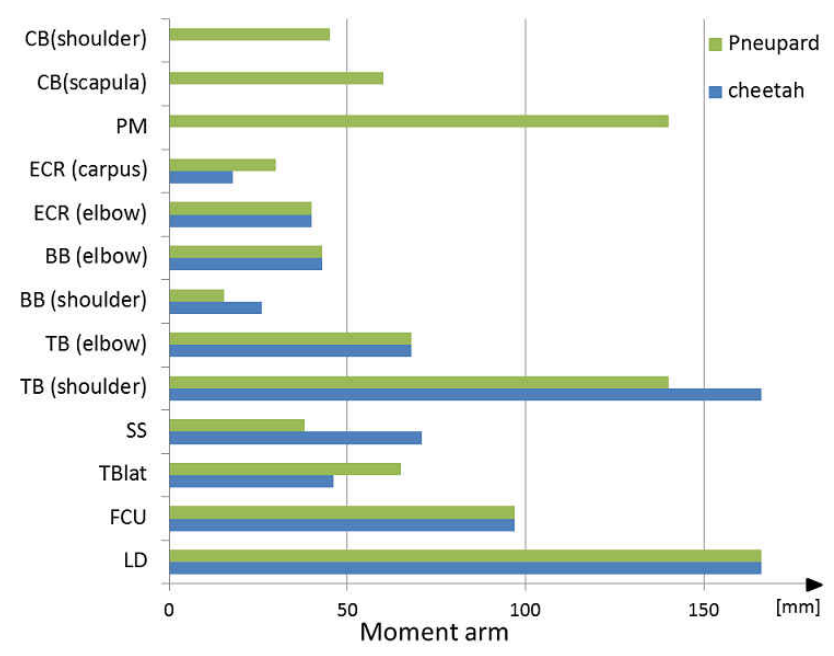

Fig. 4. Graph of comparing the muscles' maximum moment arm (Pneupard) with that of a cheetah. To the best of the author's knowledge, the moment arm of clavo brachialis (CB) and pectoralis major (PM) are not mentioned anywhere. We determine these moment arms as take account of muscle attachment or range of motion of joints.

muscle activity pattern for each phase. Touchdown is initiated when all muscles pass a preset value: supraspinatus (SS), clavo brachialis (CB) and triceps brachii (lateral and medial) (TBlat) are activated during this phase. Stance is initiated when the foot makes contact with the ground: supraspinatus (SS), pectoralis major (PM), flexor carpi ulnaris (FCU), latissimus dorsi (LD), triceps brachii (long) (TB), triceps brachii (lateral and medeal) (TBlat) are activated during this phase. Liftoff commences when the force of the sole sensor is sufficiently low: supraspinatus (SS), flexor carpi ulnaris (FCU), latissimus dorsi (LD), triceps brachii (long) (TB) and triceps brachii (lateral and medial) (TBlat) are activated in this phase. Swing commences as soon as the foot leaves the ground, and is produced by activation of clavo brachialis (CB) and biceps brachii (BB). The transition between the

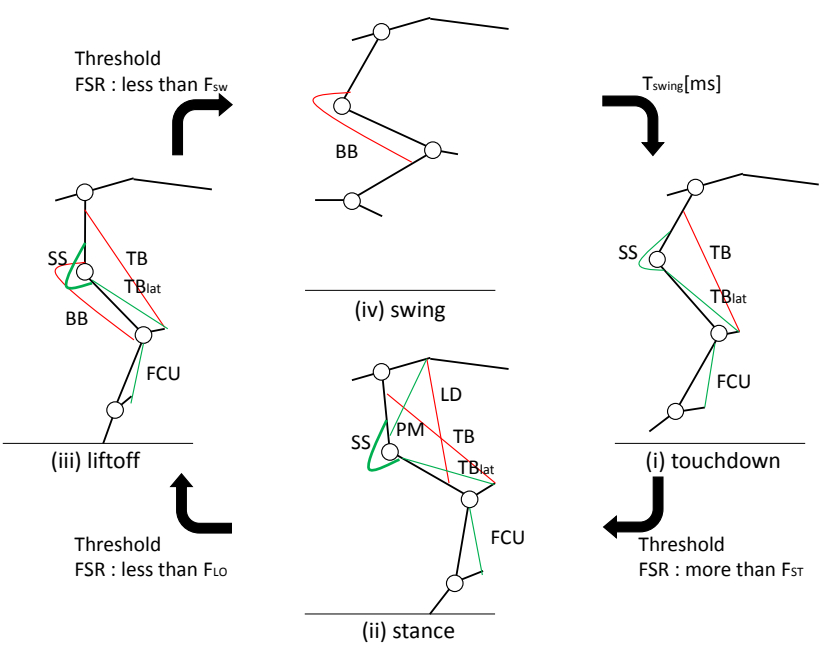

Fig. 5. Phase transition for walking action of the forelimb. The Touchdown phase (i) is the term for initiating the pneumatic muscles' pressure and joint angles. Starting from the Touchdown phase (i), the phase is changed to the Stance phase(ii) when the force of the sole exceeds $F_{S T}[\mathrm{~N}]$. The phase is changed to Liftoff phase (iii) when the force of the sole falls below $F_{L O}[\mathrm{~N}]$. The phase is changed to Swing phase (iv) when the force of the sole falls below $F_{S W}[\mathrm{~N}]$. The phase is changed to Touchdown phase when $T_{\text {swing }}[\mathrm{ms}]$ has passed.

touchdown and stance, stance and liftoff, and liftoff and swing phases is triggered by a sole sensor. Being $F[\mathrm{~N}]$ the ground reaction force resistered on the tip of the paw, $F_{S T}, F_{L O}$ and $F_{S W}[\mathrm{~N}]$ are the thresholds for changing the phases to the next. Each $F[\mathrm{~N}]$ are defined by trial-and-error method in advance. The transition of the swing-touchdown phase takes place with timing control. The right and left legs are totally uncoupled, and are independent of each other's state.

\section{EXPERIMENT AND RESULT}

We are able to perform the walking experiment involving Pneupard's forelimb on a treadmill. Forelimbs are bioinspired, but hindlimbs are simulated by a sliding strut and have no DOF. The sliding strut is fixed to the guiding rails, which prevents it from changing position. Upon setting a constant speed for the treadmill, the forelimbs are able to perform stable walking motion.

In Fig. 6, we show the activation pattern as a function of pressure for 7 pneumatic artificial muscles. This activation pattern shows the target pressures of each muscle in each walking phase. This pattern is based on data from EMG signals that are extracted from cats while walking. We use compressed air, which is at a high pressure of $0.6 \mathrm{MPa}$, for activate the pneumatic artificial muscles. When a muscle is activated, the pressure of a muscle is $\pm 0.125 \mathrm{MPa}$ from the target pressure. According to the biological muscle pattern, during the walking experience, we evaluate the biological characteristics of our designed robot. Thresholds of the force of the sole, $F_{S T}, F_{L O}$ and $F_{S W}$ are about 0.10,19.6 and $9.8[\mathrm{~N}]$ each.

Fig. 7 shows snapshot of the robot performing a walking 


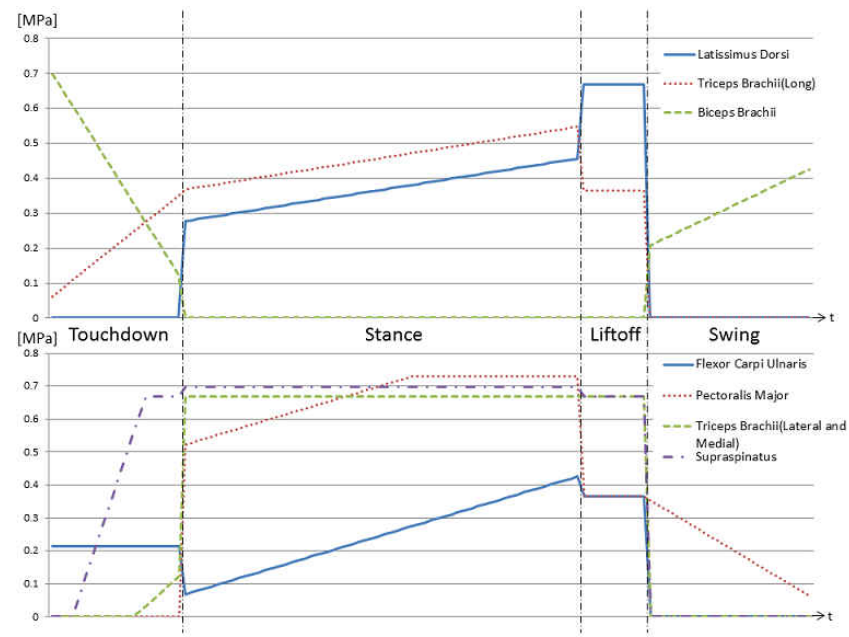

Fig. 6. The activity pattern of each muscle. The upper graph shows activity patterns of the bi-articular muscles (triceps brachii (long), biceps brachii, and latissimus dorsi). The lower graph shows activity patterns of the mono-articular muscles (supraspinatus, triceps brachii (lateral and medial), pectoralis major, and flexor carpi ulnaris).

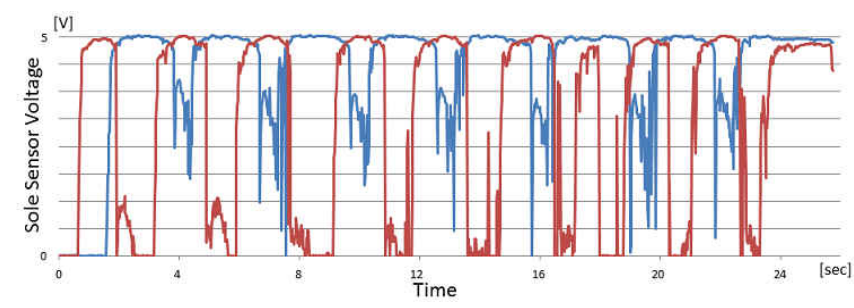

Fig. 8. The graph of sole sensor voltage transition. The left forelimb is depicted in red, while the right is depicted in blue. When the sensor voltage is high, the forelimb touches the ground. In contrast, when the sensor voltage is low, the forelimb does not touch the ground. As seen from the diagram, the right and left legs alternately touch the ground.

motion. The treadmill runs at a constant speed of $0.72 \mathrm{~km}$ $\mathrm{h}^{-1}$. One walking cycle lasts for about $3 \mathrm{sec}$. Fig. 8 shows the sole sensors' voltage transition for approximately 16 steps. The blue line represents the right leg's data, while the other is for the left leg. When the sensor's voltage is high, the leg touches the ground (stance and liftoff phase): when the sensor's voltage is low, the leg does not touch the ground (swing and touchdown phase). Although there are few exceptions, we can observe that the right and left leg alternately touch the ground.

Fig. 9 shows the gait diagram and phase contrast of each step. This graph is a stable evaluation graph of the walking motion obtained by Ekeberg et al. [16]. The phase contrast of each step is defined by:

$$
\Phi_{n}=\frac{T_{n}-T_{n-1}}{T_{n+1}-T_{n-1}}
$$

where $\Phi_{n}$ is the phase contrast and $T_{n}$ is the time for which the forelimb touches the floor at the $n$-th step. The phase contrast of each forelimb is defined in association with the timing of the floor touching for the opposite forelimb.

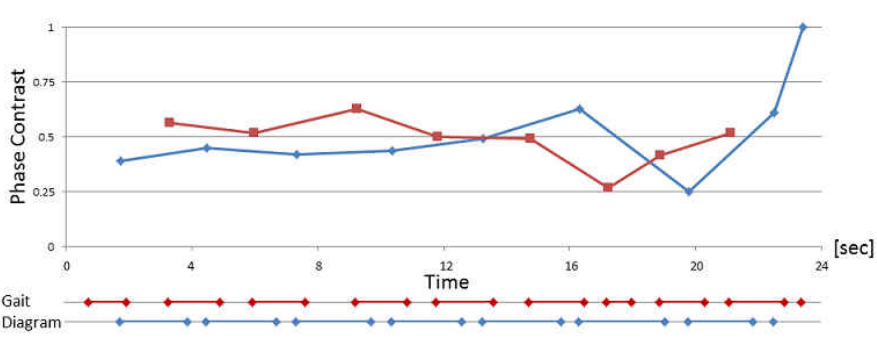

Fig. 9. The gait diagram and the phase contrast for right and left legs. The left forelimb is depicted in red, while the right is depicted in blue. For the gait pattern diagram, the leg touches the ground as indicated by the existing lines. The phase contrast of each leg is defined in accordance with the floor touching timing of the opposite leg.

\section{DISCUSSION}

In this paper, we produce the forelimb of a bio-inspired quadruped robot-modeled cheetah and propose a sole reflexbased control that treats walking as a sequence of 4 phases: touchdown, stance, liftoff, and swing. Then, we make the forelimb walk on a treadmill. During experimentation, we connect the hindlimb to guide rails, because the forelimb leans forward and cannot walk well when the hindlimb movement is not constrained using the guide rails: the forelimb falls forward when it performs a thrusting motion since there is no support in front of it. We believe that the reason for which quadruped animals do not fall forward may be because the forelimb moves in tune with the speed of advance.

Fig. 8 shows that the right and left legs alternately touch the ground without exception. Pneupard's forelimb can walk 16 steps on a treadmill. At the 6-th step of the right leg, the left leg finishes one cycle (touchdown-stance-liftoff-swing) when the right leg is still in stance phase. At that time, the left leg had not completely touched the ground, and it appeared that the left leg was slipping. We believe that this is because the robot's weight is placed on the right leg. Because of the deviation of the weight, the left leg could not sufficiently touch the ground. From another point of view, in spite of a leg slipping, the forelimb is able to continue walking a little. In other words, it indicates that our designed forelimb may walk even with varying friction of the ground is for the right and left legs.

Fig. 9 shows the phase diagram and phase contrast of the forelimbs. This graph is the evaluation method of stable walking used by Ekeberg et al. [16]. In Ekeberg's work, both limbs had a phase contrast of 0.5 when walking stably. We believe that this is because a computer simulation can produce perfectly symmetric muscles and links in an ideal simulation environment. In this figure, the phase contrast of our forelimbs is also about 0.5. Just before the robot fell forward, the numerical value of the phase contrast is different from 0.5. Compared with a computer simulation, real environments possess many elements which change dynamically. Additionally, there are small differences,e.g., the lengths of links, internal pressures in pneumatic artificial muscles, and so on, which is why our robot falls forward, 


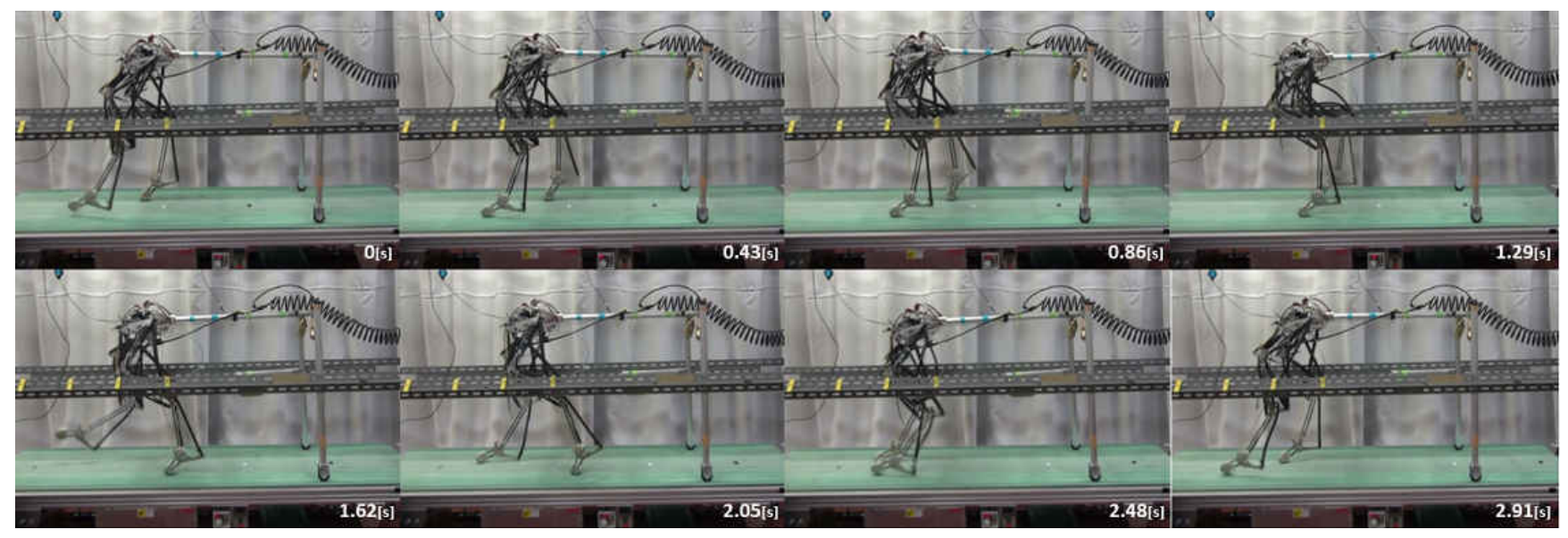

Fig. 7. Snapshot of the robot walking on a treadmill. One walking step takes about 3 seconds. This shows the procession of one walking step cycle for the left forelimb from the touchdown phase to the swing phase.

even though the phase contrast of both the legs is 0.5 .

Finally, in Fig. 7, we observe the experimental assembly walking on a treadmill. Guide rails were installed on both sides of the treadmill to prevent the robot from falling sideways, and hindlimbs were connected to them. This snapshot shows the procession of one walking step from the touchdown phase to the swing phase.

Based on these results and studies, we believe that Pneupard's forelimb has many biological features. Our robot can walk as a result of interaction between the body, a simple control system, and the peripheral environment.

In the future, we plan to connect the spine and hindlimbs to the forelimbs. First, we will connect the hindlimbs to forelimbs via the spine, which has no DOF (Fig. 10). Second, we will give the spine a few DOF. In addition, we want to explore the muscle's contribution to stable walking while reproducing quadrupedal walking. In the future, we believe that it will be possible to investigate the gait transition using a Pneupard model. As a long term view, we believe that this research will contribute to the development of robotic technologies and investigation of animals' high adaptive mechanisms.

\section{REFERENCES}

[1] George E. Goslow, Robert M. Reinking JR., and Douglas G. Stuart. The cat step cycle: Hind limb joint angles and muscle lengths during unrestrained locomotion. volume 141, pages 1-42, 2005.

[2] S.Miller, J. Van Der Burg, and F.G.A Van Der Meche. Locomotion in the cat : basic programms of movement. volume 91, pages 239-253, 1975.

[3] S.Miller and F.G.A.Van Der Meche. Movements of the forelimbs of the cat during stepping on a treadmill. volume 291, pages 255-269, Jun 1975.

[4] Arthur William English. Functional analysis of the shoulder gridle of cats during locomotion. volume 156, pages 279-292, May 1997.

[5] Arthur William English. An electromygraphic analysis of forelimb muscles during overground stepping in the cat. volume 76 , pages 105-122, October 1978.

[6] Patrick J. Whelan. Control of locomotion in the decerebrate cat. Progress in Neurobiology, 49:481-515, April 1996.

[7] Rodney A. Brooks. Intelligence without representation. Artificial Intelligence, September 1987.

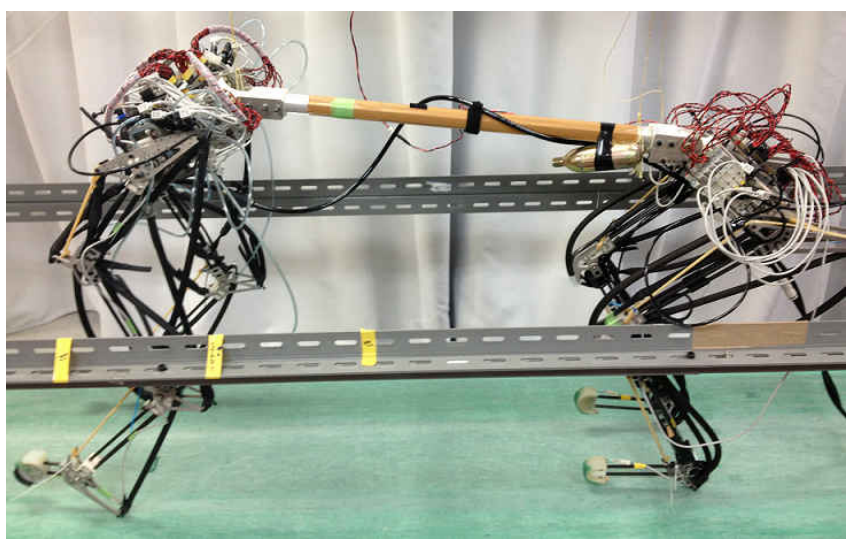

Fig. 10. The design of Pneupard modeled cheetah. The robot is constructed using forelimbs as introduced in this paper, and hindlimbs as per our previous papers [18][19]. The spine has no DOF, and can be regarded as one rigid body.

[8] Koichi Osuka. From model based control to dynamics based control. 'explicit model' and 'implicit model' in robotics. Institute of System, Control and Information Engineers, 43(2):94-100, Feb. 1999.

[9] Koichi Osuka. Implicit control and exploit control in intelligent motion. 62(3):47-51, 2010.

[10] Satoshi Ito, Hideo Yuasa, and Koji Ito. Oscillator-mechanical model of the pattern transition on quadrupedal locomotion based on energy expenditure. T.SICE, 32(11):1535-1543, Nov. 1996.

[11] Marc H. Raibert. Legged Robots That Balance. The MIT Press, 1986.

[12] Fumiya Iida and Rolf Pfeifer. "cheap" rapid locomotion of a quadruped robot: Self-stabilization of bounding gait. Proceedings of Intelligent Autonomous Systems, (8):642-694, 2004.

[13] Yoshiyuki Sakai, Tomoki Sato, Dai Owaki, and Akio Ishiguro. Realization of stable quadruped gait transition by changing body stiffness. Proceedings of the 2007 JSME Conference on Robotics and Mechatronics, 7(2):2A1-A10(1).

[14] Katsuyoshi Tsujita, Toshiya Kobayashi, Takashi Inoura, and Tatsuya Masuda. Gait transition by tuning muscle tones using pneumatic actuators in quadruped locomotion. pages 22-26, 2008.

[15] Yasunori Yamada, Satoshi Nishikawa, Kazuya Shida, Ryuma Niiyama, and Yasuo Kuniyoshi. Neural-body coupling for emergent locomotion: A musculoskeletal quadruped robot with spinobulbar model. In IROS'11, pages 1499-1506, 2011.

[16] Orjan Ekeberg and Keir Pearson. Computer simulation of stepping in the hind legs of the cat: An examination of mechanisms regulating the stance-to-swing transition. Neurophysiology, 94:4256-4268, 2005. 
[17] Andre Rosendo, Kenichii Narioka, and Koh Hosoda. Muscle roles on directional change during hopping of a biomimetic feline hindlimb. pages 1050-1055, December 2012.

[18] Shogo Nakatsu, Andre Rosendo, Kenichi Narioka, and Koh Hosoda. Reflective walking of a bio-inspired quadruped robot. May 2013.

[19] Andre Rosendo, Shogo Nakatsu, Kenichii Narioka, and Koh Hosoda. Pneupard: A biomimetic musculoskeletal approach for a felineinspired quadruped robot. November 2013.

[20] Penny E. Hudson, Sandra A. Corr, Rachel C. Payne-Davis, Sinead N. Clancy, Emily Lane, and Alan M. Wilson. Functional anatomy of the cheetah (acinonyx jubatus) forelimb. volume 218, pages 375-385, 2011.

[21] Kurt Stephen Aschenbeck. Design of a quadruped robot driven by air muscles. Case Western Reserve University, Mar. 2006. Mechanical and Aerospace Engineering. 